\title{
Message from the past president of the American Hernia Society
}

\author{
M. E. Arregui
}

(C) Springer-Verlag 2009

Hernia surgery is one of the most common general surgical procedures performed in the United States and globally. Worldwide, approximately 20 million inguinal hernias are repaired annually. In China, 2,000,000 inguinal hernias are diagnosed every year. It is estimated that about 18,000 surgeons in the United States routinely perform hernia repairs. Approximately 1,000,000 hernia repairs are performed in the United States every year. About 800,000 of these repairs are inguinal hernias. Due to the proliferation of improved techniques, advances in biomaterial and the need for better education on these advances in hernia repair for both the practicing surgeon and surgeon in training, the American Hernia Society (AHS) was started in 1997. The mission of the American Hernia Society is to provide a professional forum for the exchange of information and education regarding historic, current and future methods of diagnosis and treatment of abdominal wall abnormalities. Since its inception, the AHS has grown to over 1,000 members.

Last year's meeting in Scottsdale, Arizona was an outstanding program with surgeons from North, Central and South America attending. Internationally, 20 countries were represented. The program was lively, featuring not only expert presentations on inguinal and ventral hernia but on complications including chronic pain and medico legal

M. E. Arregui: Past President of the American Hernia Society, 2008-2009.

M. E. Arregui $(\bowtie)$

Department of General Surgery,

St. Vincent Hospital, 4802 Harcourt Road,

Suite 815, Indianapolis, IN 46260, USA

e-mail: arregui@ameritech.net issues. This program featured the Great Mesh Debate before a standing room only crowd. Entries for awards on best papers and for resident and fellow awards were highly competitive.

This year, September 9-12 in Berlin, Germany, the fourth joint hernia congress was co-hosted by the American Hernia Society and the European Hernia Society with invited participation of the Asia-Pacific Hernia society. This meeting highlighted the global interest in hernia surgery. It also provided a tremendous opportunity to share ideas on the management of this complex problem from different perspectives.

Next year, the American Hernia Society congress will be in Orlando, Florida, on March 17-20 at the JW Marriott Grande Lakes. This meeting, chaired by Dr. John Murphy, will continue to provide the most complete update on hernia surgery. Information on this meeting can be found at www.americanherniasociety.org.

These past 2 years have been busy for the AHS. The demand for information on hernia repair and educational techniques continues to grow. AHS will continue to provide the forum for education and research in its annual meeting. This will include not only didactic sessions and lively, controversial debates but also clinical as well as basic research as oral, posters and video presentations by practicing surgeons, Residents and Fellows. Global views from the American continent and other continents as well as academic and private surgeons are presented. Beyond the annual meeting, to continue communication with members and provide ongoing access to education, research and product information, the AHS is upgrading its web site. The upgrade will be more user-friendly with the capability to provide more member services including easy access to the official journal of the AHS and ultimately to provide guidelines, educational articles, video presentations and more. 
Hernia, the world journal of hernia and abdominal wall, started in 1997 by the American Hernia Society and European Hernia Society is the official journal of the AHS. It is provided to all active members of the AHS.

The AHS Foundation, chaired by Dr. Karl LeBlanc, has been hard at work-raising funds for research, educational and charitable projects promoted by the AHS. The foundation has provided support for Dr. Chuck Filipi and fellow AHS surgeons to perform hernia repairs in the Dominican Republic.

This year, out of over 40 applicants, the AHS selected Dr. Thomas Satterwhite as its first recipient for 2009 Hernia Resident/Fellow research grant from Life Cell Corporation. His research project is given as follows: complex abdominal wall reconstruction using bioprosthetic materials and component separation: The sandwich herniorrhaphy. This is one of the benefits the members of the AHS have received due to our relationships to medical industry through the Corporate Alliance.

This is truly a great re-structuring time for the AHS as it proceeds to the next level of a maturing surgical society dedicated to the art and science of hernia surgery with the goal of advancing clinical and basic research, disseminating current knowledge and providing teaching to practitioners at all levels from residency to private practice and university surgeons. We would like to thank all the AHS members from North, Central and South America, Corporate Alliance and our colleagues from the European Hernia Society who have helped realize the mission of the AHS.

It has been my privilege to serve as President of the American Hernia society this past year and wish our current President, Enrico Nicolo, well in the rewarding task of advancing our great society. 